\title{
Benchmark results in the 2D lattice Thirring model with a chemical potential
}

\author{
Venkitesh Ayyar, ${ }^{1}$ Shailesh Chandrasekharan, ${ }^{2}$ and Jarno Rantaharju ${ }^{2}$ \\ ${ }^{1}$ Department of Physics, University of Colorado, Boulder, Colorado 80309, USA \\ ${ }^{2}$ Department of Physics, Duke University, Box 90305, Durham, North Carolina 27708, USA
}

(Received 15 December 2017; published 5 March 2018)

\begin{abstract}
We study the two-dimensional lattice Thirring model in the presence of a fermion chemical potential. Our model is asymptotically free and contains massive fermions that mimic a baryon and light bosons that mimic pions. Hence, it is a useful toy model for QCD, especially since it, too, suffers from a sign problem in the auxiliary field formulation in the presence of a fermion chemical potential. In this work, we formulate the model in both the world line and fermion-bag representations and show that the sign problem can be completely eliminated with open boundary conditions when the fermions are massless. Hence, we are able accurately compute a variety of interesting quantities in the model, and these results could provide benchmarks for other methods that are being developed to solve the sign problem in QCD.
\end{abstract}

DOI: 10.1103/PhysRevD.97.054501

\section{INTRODUCTION}

Traditional lattice calculations of quantum field theories often encounter sign problems in the presence of a chemical potential. An excellent example is QCD, in which it is impossible to accurately compute quantities at a nonzero baryon density, especially at low temperatures [1]. Over the past decade, ideas like the complex Langevin approach [2] and the Leftchetz thimble approach [3] have been proposed as potential solutions to sign problems including QCD. When these methods are tested on simple models in which exact results are available [4-6], we not only find potential pitfalls of the methods but also learn new directions to avoid them [7-9]. While these ideas have also been able to capture some of the qualitative features of more complex field theories [10,11], in these cases, the numerical results are not always compared with benchmark calculations obtained with other methods in which the errors can be controlled. An exception to this has been studies of bosonic field theories at finite densities in which a controlled Monte Carlo algorithm in the world line representation free of sign problems is available $[12,13]$. Producing such benchmark calculations that truly test the method, especially in fermionic quantum field theories with a sign problem and similar to QCD in other aspects, would be helpful and is the main motivation behind our work.

Recently, the Lefshetz thimble program got a boost when it was shown that it may be possible to use holomorphic

Published by the American Physical Society under the terms of the Creative Commons Attribution 4.0 International license. Further distribution of this work must maintain attribution to the author(s) and the published article's title, journal citation, and DOI. Funded by SCOAP . flow in complex field space to sample multiple thimbles rather than perform calculations on a single thimble as was done in the past [14]. The focus has also turned to lattice Thirring models as a prototype example of the physics of QCD [14,15]. This model has also been studied earlier in higher dimensions using stochastic quantization [16]. Also, the recent work has computed the average fermion number $\langle N\rangle$ on small but fixed spatial size $L_{X}$ as a function of the chemical potential, which is much more sensitive to the important physical scales in the problem, as compared to local densities on large space-time lattices. As shown schematically in Fig. 1, at low temperatures (or large $L_{t}$ ), the plot of $\langle N\rangle$ as a function of the chemical potential $\mu$ is expected to show a series of jumps at critical values of

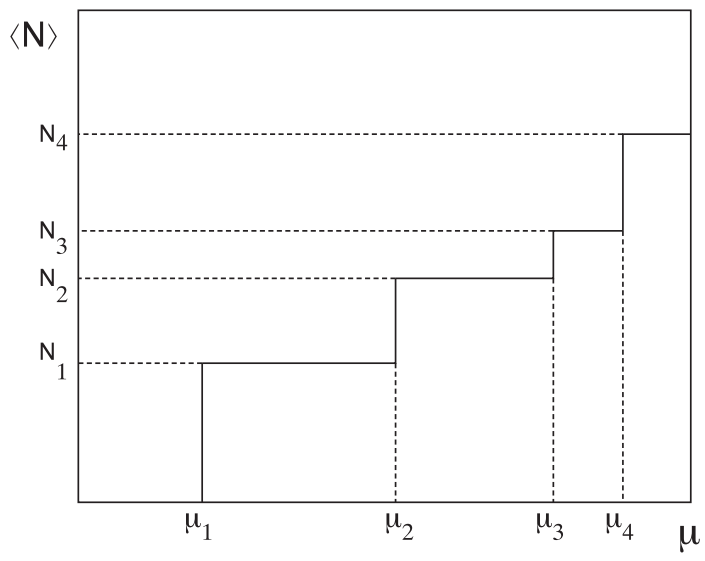

FIG. 1. A schematic plot of the particle number as a function of chemical potential for a fixed spatial size. We propose that the values of $\mu_{i}$ and the corresponding $N_{i}$ 's are easily calculable and can be used as benchmark quantities to validate a method that claims to solve a sign problem. 
the chemical potential, say $\mu_{1}, \mu_{2}, \ldots$, where the average particle number jumps to $N_{1}, N_{2}, \ldots$. The values of $\mu_{i}$ 's and $N_{i}$ 's are related to the physical scales of the problem like binding energies and scattering lengths $[17,18]$. Calculations of $\mu_{i}$ and $N_{i}$ should become harder to compute due to sign problems, especially as $\mu_{i}$ 's become large. Thus, the ability to accurately measure these quantities can be considered as a true benchmark for methods that are designed to solve sign problems. Encouraged by the fact that some of these quantitative features may be within reach, recently efforts have turned toward speeding up the calculations on larger lattices using machine learning algorithms [19]. It would indeed be exciting if this program is successful.

The motivation for our work is to help this program by accurately computing the $\mu_{i}$ 's and $N_{i}$ 's for a specific twodimensional lattice Thirring model constructed with staggered fermions. Our model is asymptotically free, and a continuum limit can be defined at zero coupling. At nonzero couplings (finite lattice spacing), the fermion in the theory is massive and mimics a baryon, while bosonic excitations made with fermion-antifermion pairs are massless and mimic pions. Thus, the similarities of our model with QCD are striking. Of course, the ground state does not break any symmetries, and the pions are not really Goldstone bosons, as was explained by Witten long ago [20], but the fermion mass generation is dynamical like in QCD, and from the point of view of sign problems, the bosons being lighter than the fermion is also similar to QCD. Interestingly, we can solve the model in both the fermion world line method and the fermion-bag approach. In the world line approach, we argue in this work that the sign problem is absent with open boundary conditions and zero fermion mass. Thus, in this limit, we are able to study large lattices and can accurately compute the critical $\mu_{i}$ 's and $N_{i}$ 's. These could provide a helpful benchmark to test new ideas that claim to solve sign problems in problems similar to QCD.

Our paper is organized as follows. In Sec. II, we discuss the model we study and the various types of representations that can be used to solve it. In particular, we show why the model in the massless limit with open boundary conditions has no sign problem in the world line formulation. In Sec. III, we discuss our Monte Carlo methods, especially the worm algorithm to update the world line representation and the fermion-bag algorithm. In Sec. IV, we discuss the results we have obtained. In particular, we define the observables we measure and discuss our results in a variety of parameter ranges. We present our conclusions in Sec. V.

\section{MODEL}

The lattice action of the model we study is given by

$$
S=\sum_{x, y} \bar{\chi}_{x}\left(M_{x, y}+m \delta_{x, y}\right) \chi_{y}+U \sum_{x, \nu} \bar{\chi}_{x} \chi_{x+\nu} \bar{\chi}_{x+\nu} \chi_{x}
$$

where the matrix $M$ is the massless staggered fermion matrix defined as

$$
M_{x, y}=\sum_{\nu} \frac{\eta_{x, \nu}}{2}\left(e^{\mu \delta_{\nu, 0}} \delta_{x+\nu, y}-e^{-\mu \delta_{\nu, 0}} \delta_{x, y+\nu}\right),
$$

where $\mu$ is the chemical potential, $m$ is the fermion mass, and $\eta_{x, \mu}$ are the usual staggered phase factors $\left[\eta_{x, 0}=1\right.$ and $\left.\eta_{x, 1}=(-1)^{x_{1}}\right]$. The four-fermion coupling $U$ can be interpreted as a current-current interaction on neighboring sites, hence the name "lattice Thirring model." When $m=0$, the model contains the well-known $U(1)$ chiral symmetry of staggered fermions. In the discussion below, $L_{X}$ denotes the number of spatial sites, and $L_{T}$ denotes the number of temporal sites in our two-dimensional square lattice. Further, we always use antiperiodic boundary conditions in time but study the effects of periodic, antiperiodic, and open boundary conditions in space.

This model has a long history and has been studied extensively in three space-time dimensions in the auxiliary field formulation [21,22] and the fermion-bag approach $[23,24]$. In three dimensions, the model with $m=0$ has two phases: a weak coupling phase with massless fermions and a strong coupling phase with spontaneously broken $U(1)$ chiral symmetry, massive fermions, and light pions. These phases are separated by a second order critical point, the properties of which were studied in the earlier work. In two dimensions, this critical point moves to the origin, and the massless weak coupling phase disappears. Further, since a continuous chiral symmetry cannot break in two dimensions, the massive fermion phase becomes critical. Thus, the two-dimensional model contains massive fermions and critical bosons, in which the mass of the fermion can be used to set the lattice spacing. The continuum limit is taken by tuning $U$ toward the origin. As far as we know, these features of the two-dimensional model with $m=0$ were never studied using the Monte Carlo method even at $\mu=0$ where there is no sign problem. The similarity of the model with QCD makes it an interesting toy model for studies at nonzero chemical potential. At a large value of $m$, this was done recently in two space-time dimensions [25].

\section{A. Auxiliary field representation}

The traditional approach to solving these models is by rewriting the partition function using an auxiliary field formulation so that it can be tackled by the Hybrid Monte Carlo algorithm. More explicitly,

$$
\begin{aligned}
Z & =\int[d \bar{\chi} d \chi] e^{-S} \\
& =\int[d \bar{\chi} d \chi] \int[d A] e^{-S_{\text {aux }},}
\end{aligned}
$$

where in the last step we have introduced a compact auxiliary field $0 \leq A_{x, \nu}<2 \pi$ associated with the bonds of the lattice and the auxiliary field action 


$$
S_{\mathrm{aux}}=\sum_{x, \nu} \frac{N_{F}}{g^{2}}\left(1-\cos A_{x, \nu}\right)+\sum_{x, y} \bar{\chi}_{x}\left(\tilde{M}_{x, y}+m^{\prime} \delta_{x, y}\right) \chi_{y}
$$

is now a Gaussian in the Grassmann fields. The Dirac matrix $\tilde{M}_{x, y}$ is defined as

$$
\sum_{\nu} \frac{\eta_{x, \nu}}{2}\left(e^{i A_{x, \nu}+\mu \delta_{\nu, 0}} \delta_{x+\nu, y}-e^{-i A_{y, \nu}-\mu \delta_{\nu, 0}} \delta_{x, y+\nu}\right),
$$

and the parameters $U$ and $m$ are related to $g$ and $m^{\prime}$ through the relations

$U=0.25\left(\frac{I_{0}\left(\frac{N_{F}}{g^{2}}\right)}{I_{1}\left(\frac{N_{F}}{g^{2}}\right)}\right)^{2}-0.25, \quad m=\left(\frac{I_{0}\left(\frac{N_{F}}{g^{2}}\right)}{I_{1}\left(\frac{N_{F}}{g^{2}}\right)}\right) m^{\prime}$.

Here, $I_{0}$ and $I_{1}$ are the Bessel function and the first modified Bessel function. The sign problem in the auxiliary field representation can be traced to the fact that $\operatorname{Det}\left(\tilde{M}+m^{\prime}\right)$ does not have any symmetries and can be complex when $\mu \neq 0$, like in QCD.

\section{B. Fermion-bag representation}

Can ideas of fermion bags help solve the sign problem present in the auxiliary field approach? In this approach, we do not introduce the usual auxiliary fields but try to regroup fermion world lines differently. Unfortunately, this regrouping is not unique and needs some thought. One possible regrouping introduced earlier for the $\mu=0$ case is based on introducing a new set of variables, the dimers $d_{x, \nu}$ for nearest neighbor interactions and monomers $n_{x}$ for the mass terms [23]. This naturally emerges when we expand the Grassmann exponential of the mass and interaction terms:

$$
\begin{aligned}
Z= & \int d \bar{\chi} d \chi e^{-\sum_{x, y} \bar{\chi}_{x} M_{x, y} \chi_{y}} \\
& \times \prod_{x}\left(1-m \bar{\chi}_{x} \chi_{x}\right) \prod_{x, \nu}\left(1-U \bar{\chi}_{x} \chi_{x+\nu} \bar{\chi}_{x+\nu} \chi_{x}\right) .
\end{aligned}
$$

We then interpret the expression

$$
\left(1+m \bar{\chi}_{x} \chi_{x}\right)=\sum_{n_{x}=0,1}\left(-m \bar{\chi}_{x} \chi_{x}\right)^{n_{x}}
$$

on each site, as introducing a monomer field $[n]$ where $n_{x}=0$ takes values 0 and 1 . The mass term $\left(-m \bar{\chi}_{x} \chi_{x}\right)$ is a monomer (single site fermion bag). Similarly, the interaction term can be rewritten using a dimer field $[d]$ such that the interaction term $\left(-U \bar{\chi}_{x} \chi_{x} \bar{\chi}_{x+\nu} \chi_{x+\nu}\right)$ is the dimer (two site fermion bag). The partition function then becomes the sum over all configurations of $[n]$ and $[d]$. Because of the Grassmann nature of the fermion field, dimers and

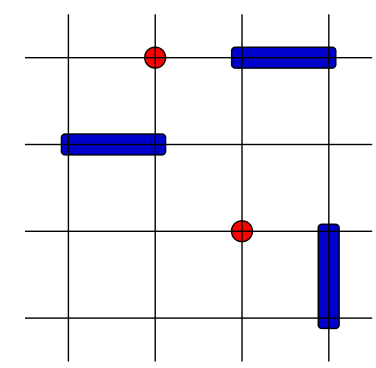

FIG. 2. An illustration of a possible configuration of dimers and monomers in a $4 \times 4$ block of the lattice. The red circles represent monomer sites, and the blue links represent dimers.

monomers cannot touch each other. Grassmann fields can be integrated over the monomer and dimer sites first, and this does not introduce any sign problems. The remaining Grassmann integral can then be performed on free sites $[f]$ that do not contain monomers or dimers. If we denote the fermion matrix $M$ restricted to the free sites as $W([f])$, we can write the partition function as

$$
Z=\sum_{[d],[n]} m^{N_{m}} U^{N_{d}} \operatorname{Det}(W([f]) .
$$

For an illustration, we show a possible configuration of dimers and monomers on a $4 \times 4$ block of lattice sites in Fig. 2. The monomers are depicted as red circles spanning a single site, and the dimers are depicted as blue links spanning two sites. The figure depicts a configuration with two free fermion bags that are isolated from each other by the dimers and monomers. Due to this, the matrix $W([f])$ is block diagonal with block matrices $W_{1}\left[f_{1}\right]$ and $W_{2}\left[f_{2}\right]$ defined within the two independent free bags. The determinant of $W([f])$ is then the product of two determinants $\operatorname{det}(W([f])=$ $\prod_{i} \operatorname{det}\left(W_{i}\left(\left[f_{i}\right]\right)\right)$.

When $\mu=0$, since the matrices $W([f])$ are always antisymmetric, $\operatorname{Det}(W([f]) \geq 0$, and the sign problem is solved. However, in the case of $\mu \neq 0$, this property no longer holds, and the determinants can be negative. This may seem surprising since in two space-time dimensions the fermion permutation sign is absent due to the fact that fermions cannot cross each other. In our model, fermions have a flavor, and they can change flavors while hopping. This is encoded in the staggered phase factors, and this leads to a sign problem. Empirically, we discovered that this remaining sign problem depends on the boundary conditions. While the sign problem is present with both periodic and antiperiodic boundary conditions, it is absent with open boundary conditions. This also means that on large space-time lattices with $L_{X}=L_{T}$ the sign problem essentially disappears, but for asymmetric lattices, it can reemerge. In the most interesting case for our studies, in which we fix the spatial lattice size $L_{X}$ and study very large values of $L_{T}$, the sign problem can become severe with periodic and antiperiodic boundary conditions. 


\section{World line representation}

In order to get a better understanding of the origin of the sign problem in our model, we look at the representation of the fermion determinant $\operatorname{Det}(W[f])$ inside free fermion bags as a sum over their world lines. This representation can be found by expanding the determinant back into the Grassmann integral form,

$$
\begin{aligned}
& \operatorname{det}(W([f])) \\
& =\prod_{x \in[f]}\left(\int d \bar{\chi}_{x} d \chi_{x}\right) e^{-\sum_{x, y \in[f]} \bar{\chi}_{x} M_{x, y} \chi_{y}} \\
& =\prod_{x \in[f]}\left(\int d \bar{\chi}_{x} d \chi_{x}\right) \prod_{x, x+\nu \in[f]} \\
& \quad \times\left(1-\frac{1}{2} \eta_{x, \nu} e^{\mu \delta_{\nu, 0}} \bar{\chi}_{x} \chi_{x+\nu}+\frac{1}{2} \eta_{x, \nu}^{\dagger} e^{-\mu \delta_{\nu, 0}} \bar{\chi}_{x+\nu} \chi_{x}\right) .
\end{aligned}
$$

This product can be represented in terms of directed fermion link variables $l_{x, \pm \nu}=0, \pm 1$, where +1 represents the term $\bar{\chi}_{x} \chi_{x \pm \nu}$ and -1 represents the term $\bar{\chi}_{x+\nu} \chi_{x}$. The determinant is replaced with a sum over all configurations of directed links.

Configurations of links only have a nonzero weight when one $\bar{\chi}$ and one $\chi$ are chosen at each site. Thus, each site must have one directed link pointing into it and one pointing out of it. The links will therefore form closed loops. In Fig. 3, we show two valid configurations with the directed links represented as arrows pointing from $\bar{\chi}$ to $\chi$. The weight of a configuration of fermion world lines is given by the product of the weights in Eq. (2.10) and a factor -1 for every closed loop arising from a reordering of $\chi_{x}$ and $\bar{\chi}_{x}$ to match the ordering of the measure,

$$
\begin{aligned}
& \operatorname{det}(W([f])) \\
& =\sum_{[l]}(-1)^{N_{\text {loops }}} \prod_{x, \alpha}\left(e^{-\mu l_{x, \alpha} \delta_{\alpha, 0}} \frac{l_{x, \alpha} \eta_{x, \alpha}}{2}\right)^{\left|l_{x, \alpha}\right|},
\end{aligned}
$$
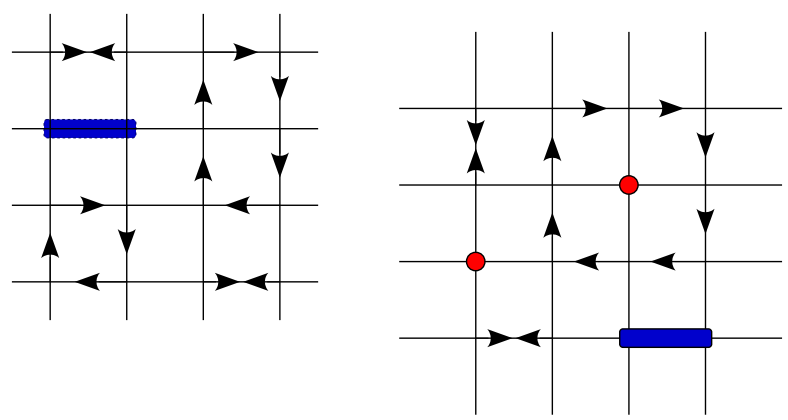

FIG. 3. Illustration of two fermion world line configurations with along with dimers and monomers. where $N_{\text {loops }}$ is the number of closed loops formed by the directed links. It is easy to verify that there are valid configurations with a negative weight. For example, the configuration on the left in Fig. 3 has a positive weight, but the configuration on the right has a negative weight.

Let us now prove that the sign problem disappears with open boundary conditions in the massless limit because configurations with a negative sign are absent at the world line level. The weight of a configuration can be written as the product of the weights of the closed loops of fermion links

$$
\begin{aligned}
& \operatorname{det}(W([f], \mu)) \\
& =\sum_{[l]} \prod_{\text {loop } \in l}\left(-\prod_{x, \alpha \in \text { loop }} e^{-\mu l_{x, \alpha} \delta_{\alpha, 0}} \frac{l_{x, \alpha} \eta_{x, \alpha}}{2}\right) .
\end{aligned}
$$

It is therefore sufficient to show that all loops that can exist in a configuration have positive weight.

The sign of a loop can be computed as a product of the four sign factors. The first sign factor $(-1)^{N_{b}}$ arises due to the fact that forward and backward links have opposite signs, where $N_{b}$ is the number of links in the loop pointing backward. The second factor $(-1)^{N_{t, \text { odd }}}$ comes from the staggered fermion phase factor $\eta_{x, 1}$, where $N_{t, \text { odd }}$ is the number of temporal links in the loop that occur on an odd spatial site. The third factor $(-1)^{W_{t}}$ comes from the antiperiodic temporal boundary conditions, where $W_{t}$ is the temporal winding of the loop. Finally, there is an extra factor of $(-1)$ for every fermion loop. This arises due to the reorganization of Grassmann variables in a closed loop before performing the Grassmann integration. Thus, the sign of the loop is given by $(-1)^{N_{s}}$, where

$$
N_{s}=N_{b}+N_{t, \text { odd }}+W_{t}+1 .
$$

In the following discussion, we will focus on open spatial boundary conditions where only three distinct topological loops are allowed, namely, $W_{t}=0,1,-1$.

Clearly, the parity of $N_{s}$ determines the sign of the loop; when it is even, the loops are positive, and when it is odd, they are negative. Let us now see why massless staggered fermions with open spatial boundary conditions can only contain loops with an even $N_{s}$. Let us define the parity of a space-time region as +1 if it contains an even number of lattice sites and -1 otherwise. The total number of sites in space-time is always even in a staggered fermion formulation. In the absence of a mass term, every loop by itself always has an even number of sites associated with it and divides space-time into two regions such that the combined number of sites of the regions is also even. However, some loops divide space-time such that each side has an odd number of lattice sites. We will argue below that the parity of regions on either side of the loop is equal to the parity of $N_{s}$ associated with the loop. This implies that loops with an 
odd value of $N_{s}$ cannot be generated because that means space-time regions with odd parity have been created. In the massless limit, such space-time regions are not allowed since they cannot be filled with loops, all of which have an even number of sites. For example, in the $W_{t}=0$ sector, loops with an odd $N_{s}$ will enclose an odd parity region, which is impossible. In the $W_{t}= \pm 1$ sectors, we can focus on loops closest to the open boundary with an odd value of $N_{s}$. Then, the space-time region connected to the boundary will contain an odd number of sites which is again impossible. Hence, loops with an odd value of $N_{s}$ are not allowed.

We still have to prove that the parity of $N_{s}$ associated with the loop is always equal to the parity of the space-time regions on either side of it. For this purpose, let us consider building an arbitrary loop from some elementary loops. It is easy to see that all loops can be built from three elementary loops:

(1) The first is the simple two site loop in the spatial direction (a spatial dimer), which has $N_{b}=1$, $N_{t, \text { odd }}=0$, and $W_{t}=0$. Hence, $N_{s}=2$, and the loop has a positive sign.

(2) The second is the temporal winding loop that goes up in time at an even site. Thus, in this case, $N_{b}=0$, $N_{t, \text { odd }}=0$, and $W_{t}=1$. Thus, again, $N_{s}=2$, and the loop is positive.

(3) The third is the reverse of the temporal loop defined above; i.e., it travels backward in time on an even site and wraps around the temporal boundary in the negative direction. Thus, $N_{b}=L_{T}, N_{t, \text { odd }}=0$, and $W_{t}=-1$. Thus, now, $N_{s}=L_{T}$, which we always take to be even. Thus, it also has a positive sign.

Note that each of these elementary loops divides space-time lattice into two regions, both of which have an even parity. Thus, we always begin with loops in which the parity of $N_{s}$ associated with the loop and the parity space-time regions on either side of it are even.

We can construct any arbitrary loop in the three topological sectors $W_{t}=0, \pm 1$ beginning with one of the elementary loops defined above in that sector and performing a series of two simple local deformations which we call the link-staple deformation and the corner-flip deformation. These are shown in Fig. 4. Let us now study the effect of each of these deformations on the value of $N_{s}$ and how they change the number of sites in the two regions on either side of the loop. We will see that these two features are correlated.

Consider first the link-staple deformations in which a link in the loop is replaced by a staple or vice versa. These deformations are depicted in Figs. 4(a) and 4(b). It is easy to verify that these deformations always change $N_{t, \text { odd }}$ by 1 and $N_{b}$ by 1 , and hence $N_{s}$ changes by an even amount. This implies that the link-staple deformations do not change the parity of $N_{s}$. Also notice that these deformations either grow the size of the loop by two sites or vice versa.

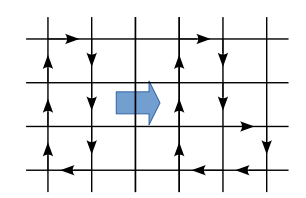

(a)

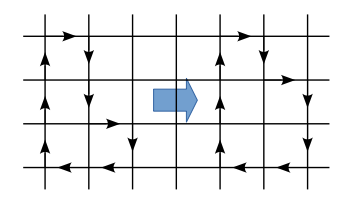

(c)

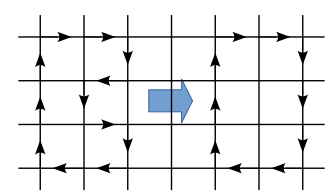

(b)

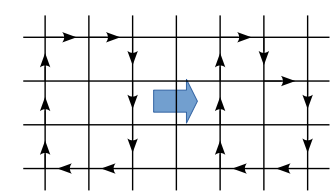

(d)
FIG. 4. Deformations that can be used to link any two loops with the same amount of spatial and temporal wrappings. In (a), a link is replaced with a staple, and in (b), a staple is replaced with a link. The sign of the loops does not change in either case. In (c) and (d), a corner is flipped. The sign changes, and the number of sites in the loop changes by 1 .

This means two lattice sites either get generated or disappear from the remaining lattice. Most importantly, both these sites appear on or disappear from the same side of the loop and so preserve the even number of lattice sites on each side.

Next, consider the corner-flip deformations in which a corner formed by the bending of the loop is flipped to the opposite site of the plaquette as shown in Figs. 4(c) and 4(d). For these deformations, only $N_{t, \text { odd }}$ changes by 1 , and hence $N_{s}$ also changes by 1 . This means corner-flip deformations do change the sign of a loop. However, notice that such deformations do not change the size of the loop but move a single lattice site from one side of the loop to the other. Thus, these deformations create loops with an odd number of lattice sites on each side.

Since we can construct all loops with these two elementary deformations acting on the three positive elementary loops, we note that loops with an odd value of $N_{s}$ will involve an odd number of corner-flip deformations and thus divide the lattice into two regions with an odd number of lattice sites on each side. Hence, as explained above, in the massless limit with open boundary conditions, loops with an odd value of $N_{s}$ are not allowed. All loops are positive.

Clearly, the above arguments fail in the presence of a nonzero mass, where single sites can be saturated with the mass term. The arguments also fail when the spatial boundary condition is not open and links or loops may cross the boundary. An example of an allowed negative signed loop is shown in Fig. 5 on the left, assuming the lattice is periodic. With periodic and antiperiodic boundary conditions, we can have other more complicated loops as shown in Fig. 5 on the right. Thus, the sign problem can be completely eliminated by open boundary conditions in the spatial direction. This feature of the world line formulation is well known and specific to two-dimensional models [26-28]. In higher dimensions, the argument for the 

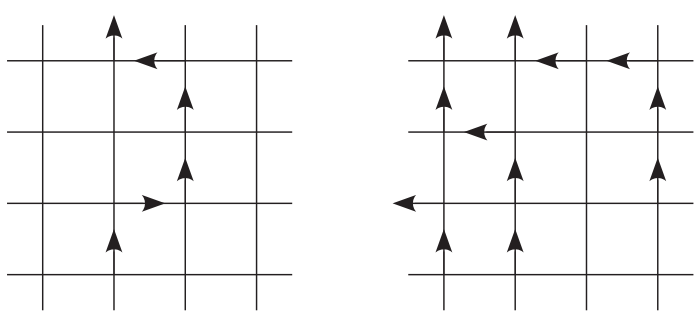

FIG. 5. The figure on the left shows a negative sign fermion loop that wraps along the temporal direction. Such a loop is generated when an odd number of sites cross the fermion world line as it is obtained through a series of deformations starting from a straight temporal loop. The figure on the right shows a loop with negative sign when the spatial boundary condition is (anti)symmetric.

positivity of all fermion world line configurations fails, and significant cancellations between world line configurations will be necessary for alleviating the sign problem. The fermion-bag approach can be helpful in this regard [25].

Before we end this section, we would like to comment on the connection between our proof for the absence of the sign problem here and the one given in Ref. [29] for the massless Schwinger model. As shown in the previous work, the presence of gauge fields allows us to absorb the staggered fermion phase factors into the gauge fields. This leads to a simple algebraic formula for the sign factor for each configuration. In the previous work, it was then argued that this sign factor is always positive. Due to the absence of gauge fields in our work, we had to deal with the staggered fermion phase factors directly. Hence, we focused on an expression for the sign coming from each loop and showed that only positive loops contribute by understanding the topology associated with space-time regions on either side of the loop. Interestingly, we can also use our topological insight and argue for the absence of the sign problem from the fermionic sector in the massless Schwinger model. The argument is based on the fact that with $U(1)$ gauge fields, fermion loops can only appear in the sectors $W_{t}=0,1,-1$. Open boundary conditions are not necessary. Clearly, $W_{t}=0$ loops will be positive for the same reason as we discuss above. Further, the $U(1)$ gauge field forces every $W_{t}=1$ loop to be accompanied by a neighboring $W_{t}=-1$ loop and vice versa. Thus, the region between these two temporal winding loops must have positive parity. This implies that every $W_{t}= \pm 1$ loop must have $N_{s}=0$ and hence will be positive. Thus, again, only positive fermion loops contribute.

\section{MONTE CARLO UPDATES}

Monte Carlo methods for updating both the world line representation and the fermion-bag representations are by now well developed [30-35]. We use a worm algorithm to update the fermion lines and dimers, using updates like the one illustrated in Fig. 6. To begin an update, we suggest

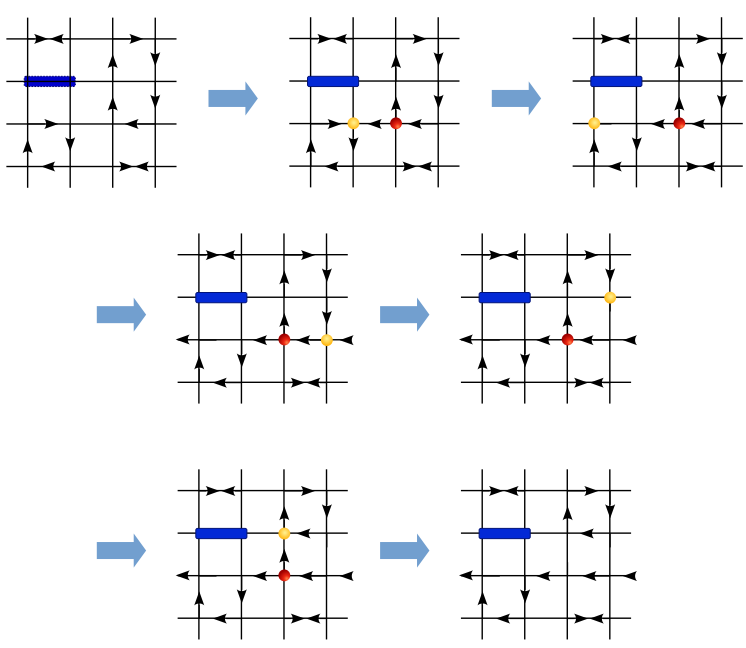

FIG. 6. An illustration of the worm update used in the world line formalism. The solid dots represent the head and the tail of the worm where the configuration has defects. At the end, the defects disappear, and a new allowed configuration is generated.

randomly changing some fermion link $l_{x, \nu}$. The update is then accepted with the absolute value of the weight given in Eq. (2.11). If the link is changed, two defects are generated in the lattice configuration, which are allowed. The defects are the head and tail of the worm. The head of the worm then propagates by updating the neighboring links. When the head returns to its tail, the worm closes, the defects disappear, and the update is complete. The various steps of how the defect propagates are shown in shown Fig. 6. The configuration of dimers may also be updated during the worm update. When this is done, we have to use the weights of including or removing a dimer. Figure 7 shows the steps for an update that changes the dimer number.

In contrast to the worm algorithm, we sample fermionbag configurations using a local Monte Carlo update that
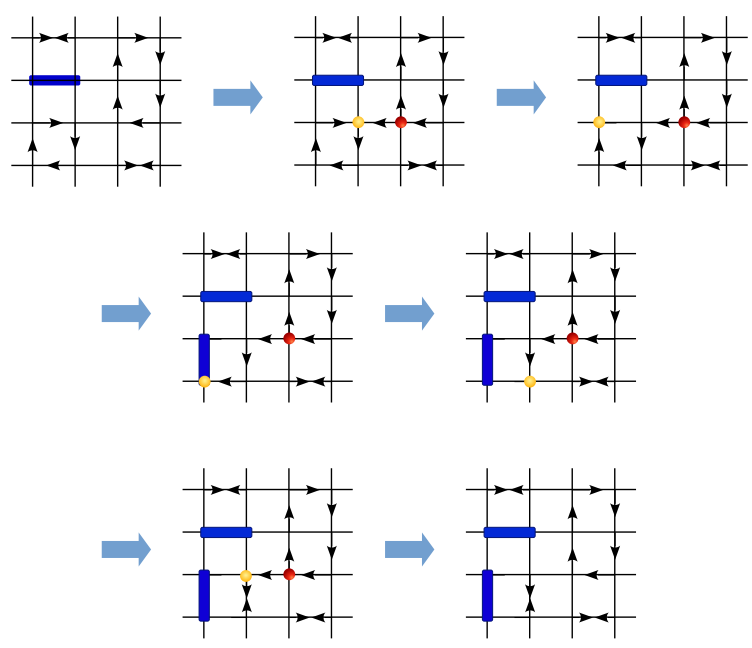

FIG. 7. An illustration of the worm update that changes the dimer number. 
involves adding or removing dimers or pairs of monomers. Each proposal is accepted with the probability

$$
P_{\text {acc }}=\frac{m^{N_{m}^{\prime}} U^{N_{d}^{\prime}} \operatorname{det}\left(W\left(\left[f^{\prime}\right], \mu\right)\right)}{m^{N_{m}} U^{N_{d}} \operatorname{det}(W([f], \mu))},
$$

where the new configuration is denoted with primed variables. The fact that one has to use ratios of fermion determinants that are nonlocal helps to reduce autocorrelation times. We also can update large regions of space-time by using a background field method used recently in Ref. [35]. The sampling is made more efficient with a move that switches the places of a monomer and a dimer if the two are on neighboring sites. Since the weights of the two configurations are the same, this update is very quick.

\section{NUMERICAL RESULTS}

In this work, we compute three observables in order to understand the physics of our model. The first is the chiral condensate susceptibility $\chi$, defined by the relation

$$
\chi=\frac{U}{V} \sum_{x, y}\left\langle\bar{\psi}_{x} \psi_{x} \bar{\psi}_{y} \psi_{y}\right\rangle .
$$

We can use it to understand the physics of bosonic excitations in our model. We also compute the chiral charge winding number susceptibility, defined by the relation

$$
\begin{gathered}
\left\langle Q_{\chi}^{2}\right\rangle=\frac{U}{V} \sum_{x \in S, y \in S^{\prime}}\left\langle J_{\alpha, x}^{\chi} J_{\alpha, y}^{\chi}\right\rangle, \\
J_{\alpha, x}^{\chi}=\frac{\epsilon_{x} \eta_{x, \alpha}}{2}\left[e^{\delta_{\alpha, 0} \mu} \bar{\psi}_{x} \psi_{x+\alpha}-e^{-\delta_{\alpha, 0} \mu} \bar{\psi}_{x+\alpha} \psi\right],
\end{gathered}
$$

where $S$ and $S^{\prime}$ are surfaces orthogonal to the direction $\alpha$. In the thermodynamic limit, the winding number susceptibility helps us understand the status of chiral symmetry as we explain below. In the world line representation, the chiral charge can be defined by the relation

$$
q_{x_{\alpha}}^{\chi}=\epsilon_{x}\left(l_{x, \alpha}+l_{x+\alpha,-\alpha}+2 d_{x, \alpha}\right),
$$

which means the susceptibility is simply

$$
\left\langle Q_{\chi}^{2}\right\rangle=\left\langle\left(\sum_{x \in \alpha} q_{\chi_{\alpha}}^{\chi}\right)^{2}\right\rangle
$$

since the chiral charge is conserved on each configuration. Finally, we measure the average fermion number using the relation $\left\langle N_{f}\right\rangle=\left\langle\sum_{x \in S} J_{0, x}\right\rangle$, where the fermion number current is given by

$$
J_{\alpha, x}=\frac{\eta_{x, \alpha}}{2}\left[e^{\delta_{\alpha, 0} \mu} \bar{\psi}_{x} \psi_{x+\alpha}-e^{-\delta_{\alpha, 0} \mu} \bar{\psi}_{x+\alpha} \psi_{x}\right]
$$

TABLE I. The scaling dimension $\nu$, chiral charge susceptibility $\left\langle Q_{\chi}^{2}\right\rangle$, and the fermion mass measured on a square lattice.

\begin{tabular}{lllc}
\hline \hline$U$ & $2-\eta$ & \multicolumn{1}{c}{$\left\langle Q_{\chi}^{2}\right\rangle$} & $m_{f}$ \\
\hline 0 & 0 & 0.25 & 0 \\
0.1 & $0.90(1)$ & $0.499(7)$ & $0.0098(4)$ \\
0.2 & $1.201(4)$ & $0.61(1)$ & $0.081(1)$ \\
0.3 & $1.303(4)$ & $0.780(8)$ & $0.183(1)$ \\
0.4 & $1.371(7)$ & $0.895(4)$ & $0.290(1)$ \\
0.5 & $1.393(3)$ & $0.972(3)$ & $0.395(3)$ \\
0.6 & $1.423(4)$ & $1.024(3)$ & $0.491(1)$ \\
1.0 & $1.467(4)$ & $1.128(2)$ & $0.793(1)$ \\
$\infty$ & 1.5 & $1.208(8)$ & $\infty$ \\
\hline \hline
\end{tabular}

and $S$ is a surface perpendicular to $\hat{t}$. In the world line representation, again, the fermion number is straightforward to calculate and is given by

$$
\left\langle N_{f}\right\rangle=\sum_{x \in S}\left\langle l_{x, \hat{t}}-l_{x+\hat{t},-\hat{t}}\right\rangle .
$$

In our definition, the fermion number is normalized to count both the Dirac and flavor degrees of freedom from a continuum limit perspective.

Using these observables, we first focus on the physics of our model at $\mu=0$ in order to bring out the similarities to QCD. As we mentioned earlier, unlike in QCD, the $U(1)$ chiral symmetry of the model cannot break in two dimensions. However, the lightest boson in the model is critical (i.e., it is massless but is not a Goldstone boson). Hence, when $L_{X}=L_{T}=L$, we expect the chiral condensate susceptibility to scale as $\chi \sim L^{2-\eta}$ for large values of $L$. The exponent $\eta$ depends on $U$ like in the usual critical phase of the two-dimensional $X Y$ model. Since the Thirring model becomes a closed packed dimer model at infinite $U$, we expect $\eta=0.5$ [36]. When $U=0$, the susceptibility diverges logarithmically with $L$, and hence $\eta=2$. Our results reproduce this and show how the exponent changes continuously between these two limits. In Table I, we give the values of $2-\eta$ obtained at various values of $U$.

In a chirally symmetric theory with massive excitations, the chiral charge winding number susceptibility $\left\langle Q_{\chi}^{2}\right\rangle$ is expected to vanish because the chiral charge cannot wind across the spatial boundaries. However, when the phase is critical like in our model, it is expected to be go to a constant in the thermodynamic limit. Our results are consistent with this expectation. The values we measured for $\left\langle Q_{\chi}^{2}\right\rangle$ at $L=256$ are given in Table I. These values are found using open boundary conditions. Further, we find that $\left\langle Q_{\chi}^{2}\right\rangle=0.25$ at $U=0$ and grows monotonically to the value of roughly 1.2 at $U=\infty$. All this is consistent with the fact that the bosonic sector of our theory is critical.

In contrast to the bosons, fermions are massive for all values of $U>0$. We compute the fermion mass $m_{f}$ as a function of $U$ using large square lattices $\left(L_{X}=L_{T}=L\right)$ as 


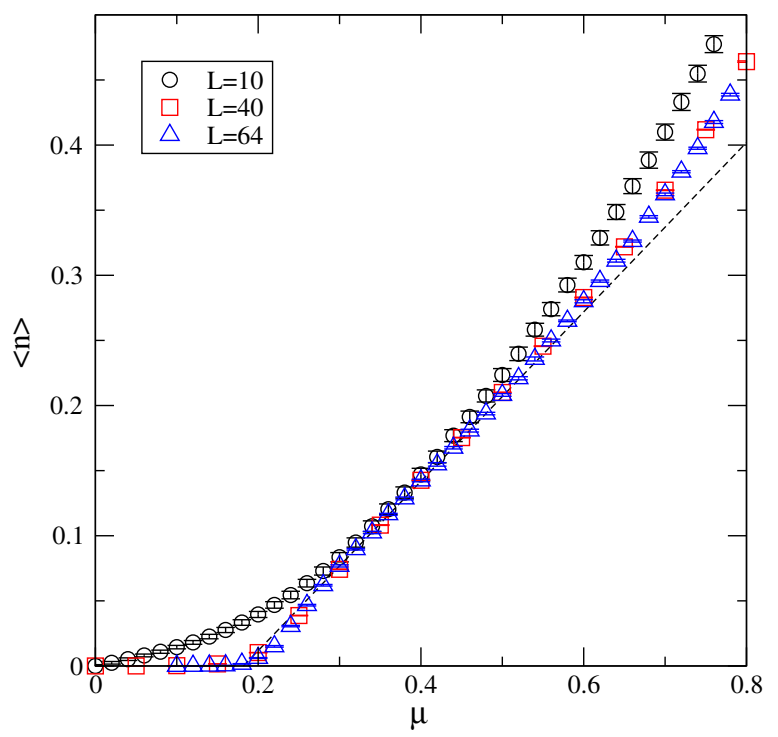

FIG. 8. The fermion number density $\langle n\rangle$ as a function of the chemical potential on a square lattice with open boundary conditions. The dashed line shows a fit to the linear region at $L=64$.

follows. In the thermodynamic limit, we expect the average fermion density $\langle n\rangle=\left\langle N_{f}\right\rangle / L_{X}$ to be zero when $\mu \leq m_{f}$ and rise linearly according to the relation

$$
\langle n\rangle=c\left(\mu-m_{f}\right)
$$

for $\mu \geq m_{f}$. This behavior should also be an excellent approximation for sufficiently large lattices. To demonstrate this, we show our results for $\langle n\rangle$ at $U=0.3$ with open boundary conditions in Fig. 8. Selected values of these data are also tabulated in Table II as a benchmark for future calculations. As we can see for $L=10$, the curve does not show the expected nonanalyticity, but for $L=40$ and $L=64$, the curves show it clearly. We can fit our data

TABLE II. Selected values of $\langle n\rangle$ plotted in Fig. 8 .

\begin{tabular}{lccccc}
\hline \hline$\mu$ & $\langle n\rangle$ & $\mu$ & $\langle n\rangle$ & $\mu$ & $\langle n\rangle$ \\
\hline \multicolumn{5}{c}{$L=10$} \\
0.16 & $0.0276(2)$ & 0.32 & $0.0948(4)$ & 0.52 & $0.2397(5)$ \\
0.20 & $0.0396(2)$ & 0.36 & $0.1204(4)$ & 0.54 & $0.2582(5)$ \\
0.24 & $0.0545(3)$ & 0.40 & $0.1472(5)$ & 0.56 & $0.2740(5)$ \\
0.28 & $0.0729(3)$ & 0.48 & $0.2074(5)$ & 0.58 & $0.2926(5)$ \\
\multicolumn{5}{c}{$L=40$} \\
0.15 & $0.0016(1)$ & 0.30 & $0.0742(4)$ & 0.45 & $0.1753(4)$ \\
0.20 & $0.0103(2)$ & 0.35 & $0.1082(4)$ & 0.50 & $0.2100(5)$ \\
0.25 & $0.0387(4)$ & 0.40 & $0.1425(4)$ & 0.55 & $0.2456(4)$ \\
\multicolumn{5}{c}{$L=64$} \\
0.16 & $0.0004(1)$ & 0.32 & $0.089(1)$ & 0.52 & $0.221(1)$ \\
0.20 & $0.0062(4)$ & 0.36 & $0.116(1)$ & 0.54 & $0.236(1)$ \\
0.24 & $0.0309(6)$ & 0.40 & $0.142(1)$ & 0.56 & $0.250(1)$ \\
0.28 & $0.0618(6)$ & 0.48 & $0.194(1)$ & 0.58 & $0.264(1)$ \\
\hline \hline
\end{tabular}

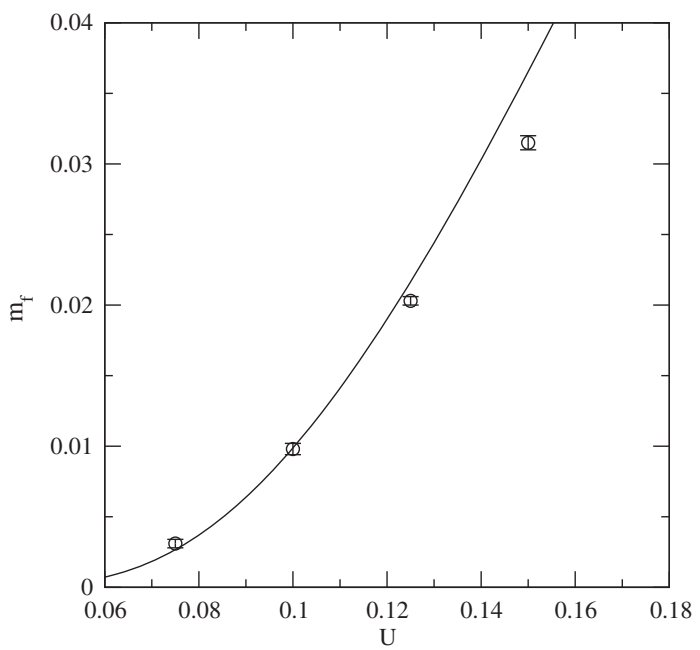

FIG. 9. Plot of the fermion mass as a function of $U$ for small values. We observe qualitatively the exponential scaling expected. The solid line is the one-loop $\beta$ function.

to the linear form which is shown as the dashed line in the fit. In Table I, we report the value of $m_{f}$ found using this method for several values of $U$. We used lattices of size of $L=64$, except for $U=0.1$, where the lattice size used was $L=128$.

The dynamical generation of fermion mass is an interesting feature of our model. While similar to the phenomenon of chiral symmetry breaking in QCD, the actual dynamical breaking of continuous symmetries is forbidden in two-dimensional models. Nevertheless, a fermion mass can be generated, and a massless boson with critical correlations can arise [20]. Finally, we note that fourfermion couplings are expected to be marginal in two dimensions, and in our case, it also happens to be marginally relevant (i.e., asymptotically free). Thus, at small $U$, the fermion mass $m_{f}$ is expected to vanish according to the relation

$$
m_{f} \approx C \exp \left(\frac{-2 \pi}{b_{0} U}\right)
$$

where $b_{0}=16$ is the one-loop coefficient of the $\beta$ function. Figure 9 shows a plot of the fermion masses at various values of $U$ obtained using our method discussed above, along with a solid line that shows the expected behavior of Eq. (4.9). For purposes of illustration, we use $C=0.49$. With these small masses, lattice volumes up to $V=1024 \times$ 1024 were necessary. It is well known that such asymptotic scaling fits do not work very well unless very large lattices are used [37]. Here, we just use the scaling to illustrate qualitatively that fermion masses do become exponentially small as $U$ becomes small.

Next, we turn to the physics of the finite chemical potential. We first consider a small spatial lattice of $L_{X}=6$ and $L_{T}=48$ and study the sign problem in the traditional auxiliary field approach with periodic boundary conditions 

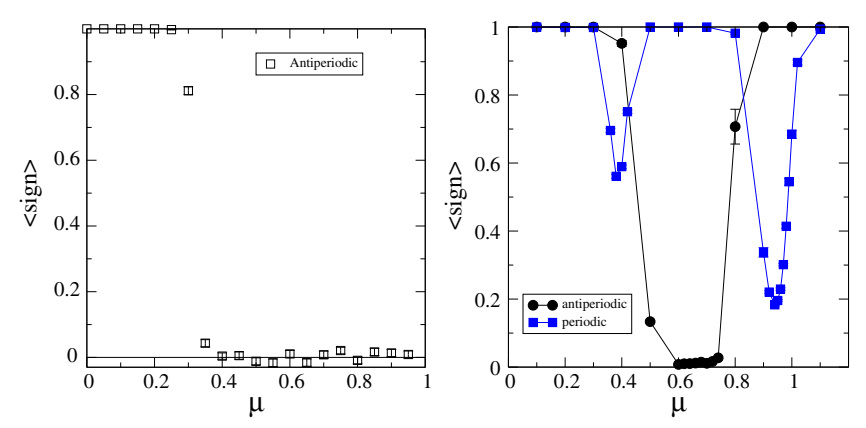

FIG. 10. The average sign of $\operatorname{det}(W)$ at $U=0.3$ as a function of the chemical potential with $L_{T}=48$ and $L_{X}=6$ in the auxiliary field representation(left) and the fermion-bag representation (right).

and compare it with the sign problem in the fermion-bag approach with both periodic and antiperiodic boundary conditions. In Fig. 10, we plot the average sign as a function of the chemical potential in the auxiliary field approach at $U=0.3$ (left) and compare it with that of the fermion-bag approach (right). We first wish to learn where the sign problem becomes severe. In the auxiliary field approach, the sign becomes severe around $\mu \approx 0.4$, while in the fermion-bag approach with antiperiodic boundary conditions, it becomes severe around $\mu \approx 0.55$. In the fermion-bag approach with periodic boundary conditions, the sign problem is never severe, although it is enhanced both at $\mu \approx 0.4$ and then again at $\mu \approx 0.9$. Can we correlate this behavior with some physics?

Let us now explore how the fermion chemical potential "dopes" the system with fermions. We again focus first on a small lattice, $L_{X}=6, L_{T}=48$ at $U=0.3$. In Table III, we present all of our results for the total fermion number as a function of the chemical potential for open (left), antiperiodic (center), and periodic boundary conditions (right). In Fig. 11, we plot these results along with the results for free fermions as solid lines. Due to the flavor degeneracy of staggered fermions, we expect all states to be at least doubly degenerate. With open periodic boundary conditions, this means all jumps must be in steps of 2 . This is what is observed. With periodic and antiperiodic boundary conditions, there is a symmetry between left- and rightmoving particles. With periodic boundary conditions, a zero momentum state is allowed which is nondegenerate; hence, the first jump in $\langle N\rangle$ near $\mu \approx 0.4$ is only by 2 . However, the second jump near $\mu \approx 0.9$ is by a factor of 4 since now nonzero momentum states are excited and each state is doubly degenerate due to the two fermion flavors. With antiperiodic boundary conditions, the lowest energy state already has momentum and hence again should have fourfold degeneracy. This is clearly seen as a jump of 4 in the free theory around $\mu \approx 0.5$. Surprisingly, in the interacting theory, this degeneracy of the lowest energy state seems to be broken. We attribute this to the fact that bound state bosons with zero momentum can emerge. The next
TABLE III. The average fermion number $\left\langle N_{f}\right\rangle$ computed at $U=0.3$ with periodic, antiperiodic, and open boundary conditions with $L_{X}=6$ and $L_{T}=48$.

\begin{tabular}{lccccccc}
\hline \hline$\mu$ & $\left\langle N_{f}\right\rangle$ & $\mu$ & $\left\langle N_{f}\right\rangle$ & $\mu$ & $\left\langle N_{f}\right\rangle$ & $\mu$ & $\left\langle N_{f}\right\rangle$ \\
\hline \multicolumn{7}{c}{ Periodic } \\
0.30 & $0(0)$ & 0.36 & $0.13(1)$ & 0.38 & $0.67(2)$ & 0.40 & $1.52(2)$ \\
0.42 & $1.91(1)$ & 0.50 & $2.0(0)$ & 0.90 & $2.36(3)$ & 0.92 & $2.87(2)$ \\
0.94 & $3.68(3)$ & 0.95 & $4.03(3)$ & 0.96 & $4.43(3)$ & 0.97 & $4.86(2)$ \\
0.98 & $5.21(2)$ & 0.99 & $5.47(2)$ & 1.00 & $5.65(2)$ & 1.10 & $5.98(2)$ \\
\multicolumn{7}{c}{ Antiperiodic } \\
0.50 & $0.03(3)$ & 0.54 & $0.19(3)$ & 0.56 & $0.56(6)$ & 0.58 & $1.17(5)$ \\
0.60 & $1.61(9)$ & 0.61 & $1.71(5)$ & 0.62 & $1.86(5)$ & 0.63 & $1.90(4)$ \\
0.64 & $1.86(4)$ & 0.65 & $1.97(2)$ & 0.66 & $1.97(2)$ & 0.67 & $2.07(2)$ \\
0.68 & $2.04(1)$ & 0.69 & $2.11(1)$ & 0.70 & $2.19(2)$ & 0.72 & $2.50(3)$ \\
0.80 & $3.95(2)$ & 0.90 & $4.00(0)$ & 1.00 & $4.27(5)$ & 1.10 & $5.94(2)$ \\
\multicolumn{7}{c}{ Open } \\
0.20 & $0.000(0)$ & 0.34 & $0.044(3)$ & 0.36 & $0.122(5)$ & 0.38 & $0.256(7)$ \\
0.42 & $0.755(8)$ & 0.44 & $0.974(8)$ & 0.46 & $1.218(7)$ & 0.48 & $1.468(8)$ \\
0.50 & $1.715(8)$ & 0.52 & $1.869(5)$ & 0.54 & $1.941(3)$ & 0.56 & $1.977(2)$ \\
0.58 & $1.992(1)$ & 0.60 & $1.996(1)$ & 0.62 & $2.000(0)$ & 0.66 & $2.008(1)$ \\
0.68 & $2.022(2)$ & 0.70 & $2.056(3)$ & 0.72 & $2.125(5)$ & 0.74 & $2.290(8)$ \\
0.80 & $3.298(6)$ & 0.82 & $3.600(9)$ & 0.84 & $3.825(6)$ & 0.86 & $3.934(4)$ \\
0.88 & $3.992(2)$ & 0.90 & $4.041(3)$ & 0.92 & $4.117(6)$ & 1.02 & $5.833(7)$ \\
1.04 & $5.937(4)$ & 1.06 & $5.975(2)$ & 1.08 & $5.991(1)$ & 1.14 & $6.000(0)$ \\
\hline \hline
\end{tabular}

momentum state is nondegenerate for $L_{X}=6$ since effectively the lattice size is halved for staggered fermions. This remains unchanged for the interacting theory as well, and two additional states are added when $\mu>1$.

Note that the first step to $\left\langle N_{f}\right\rangle=2$ for both open and periodic boundary conditions occurs around $\mu \approx 0.4$. This coincides with the point where the sign problem becomes severe in the auxiliary field approach and is somewhat enhanced in the fermion-bag approach. The sign problem in the fermion-bag approach disappears for large values of $\mu$ until around $\mu \approx 0.9$ where there is the second jump of 4 in the periodic case. The sign problem in the auxiliary field approach, on the other hand, never recovers. In the case of antiperiodic boundary conditions, the severity of the sign problem coincides with the additional plateau at $\left\langle N_{f}\right\rangle=2$ which is absent in the free theory as discussed above. While these correlations between sign problems and the underlying physics are not surprising, the fact that energies and degeneracies of the lowest lying states can be influenced by boundary conditions and interactions on small lattices offers an excellent opportunity for methods that claim to solve the sign problems to reproduce them.

Since the sign problem is absent with open boundary conditions, we can use it to study the behavior of $\left\langle N_{f}\right\rangle$ on large asymmetric lattices $\left(L_{X} \neq L_{T}\right)$ so as to understand the physics of fermion doping at a fixed $L_{X}$. One of the main results that our model shares with QCD is that fermions become massive entirely due to interaction effects and the value of the chemical potential where the first jump in $\left\langle N_{f}\right\rangle$ 

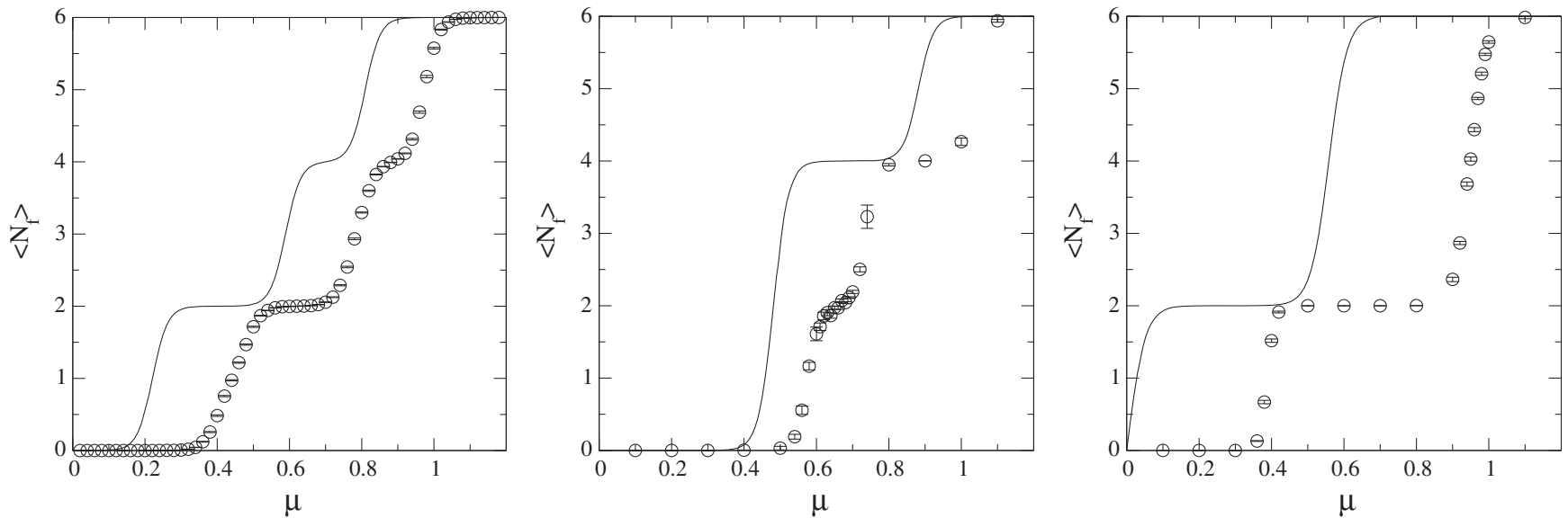

FIG. 11. The fermion number $\left\langle N_{f}\right\rangle$ at $U=0.3$ as a function of the chemical potential with open, antiperiodic, and periodic boundary conditions respectively for $L_{X}=6, L_{T}=48$. The solid line shows the value at $U=0$.
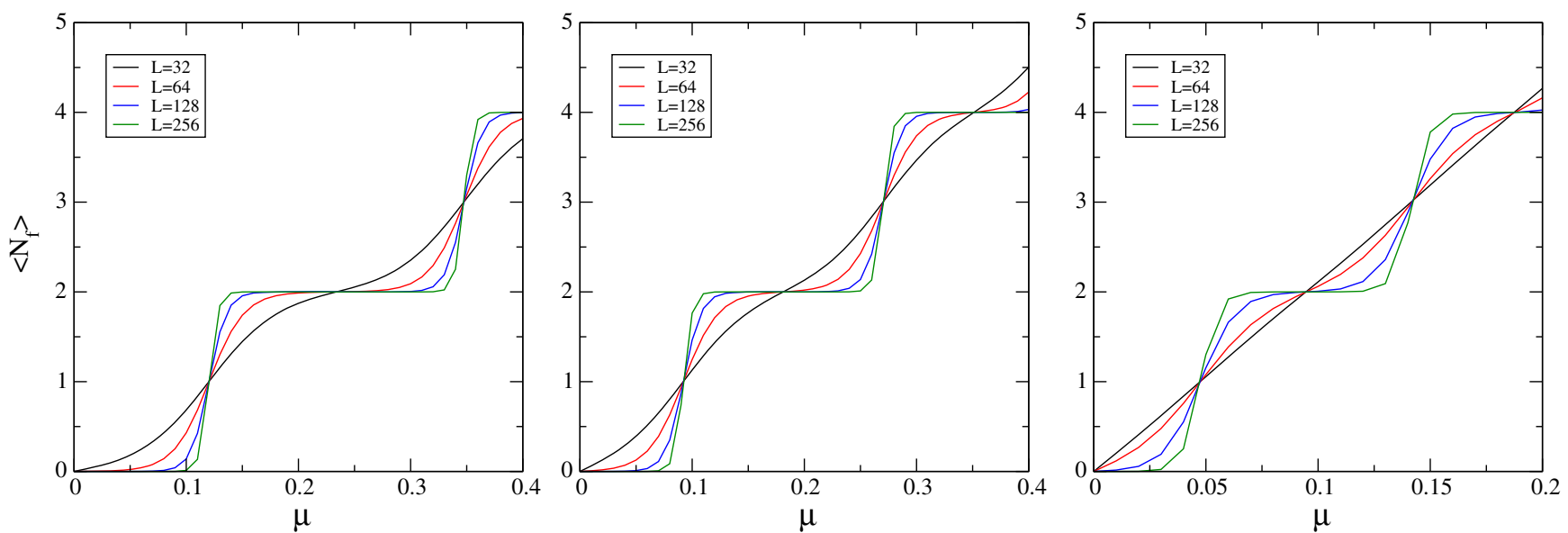

FIG. 12. The fermion number $\langle N\rangle$ with open boundary conditions at $U=0$ as a function of the chemical potential. From left to right, $L_{X}=12,16$, and 32 .
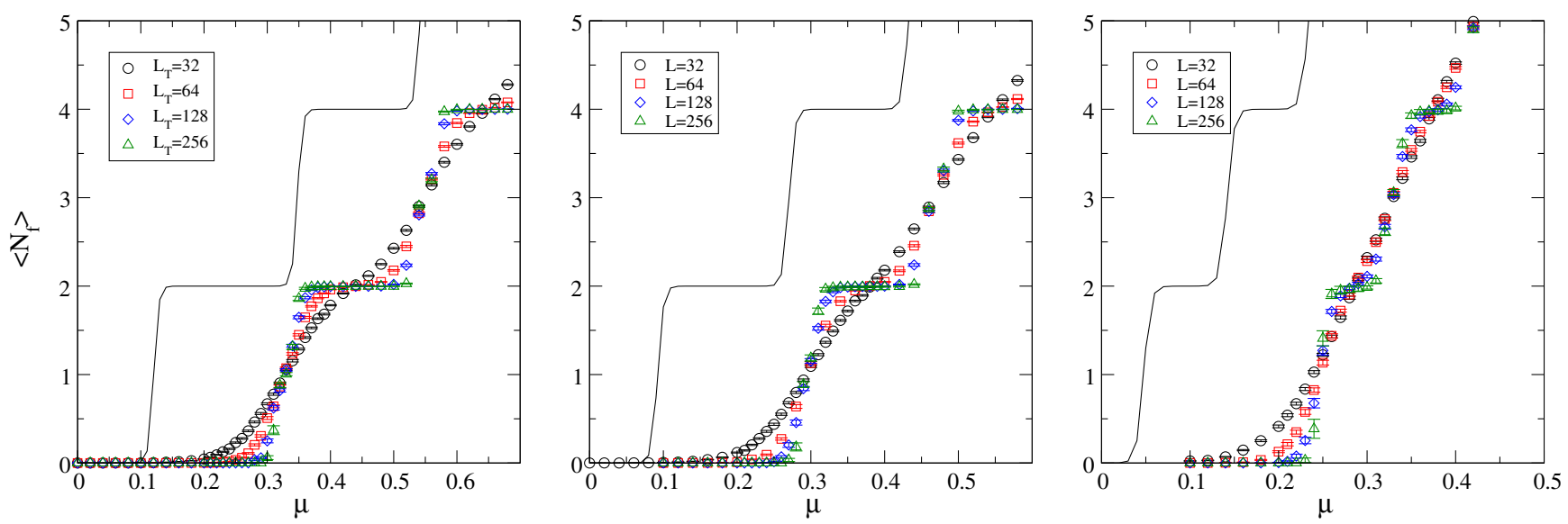

FIG. 13. The fermion number $\langle N\rangle$ with open boundary conditions at $U=0.3$ as a function of the chemical potential. The solid line shows the behavior at $U=0$ and $L_{T}=256$. From left to right, $L_{X}=12,16$, and 32 . 
TABLE IV. Monte Carlo results for $\left\langle N_{f}\right\rangle$ at $U=0.3$ with open boundaries at selected values of $\mu$ and $L_{T}$ for $L_{X}=12$. These data are plotted in Fig. 13.

\begin{tabular}{|c|c|c|c|c|c|c|c|}
\hline$\mu$ & $\left\langle N_{f}\right\rangle$ & $\mu$ & $\left\langle N_{f}\right\rangle$ & $\mu$ & $\left\langle N_{f}\right\rangle$ & $\mu$ & $\left\langle N_{f}\right\rangle$ \\
\hline \multicolumn{8}{|c|}{$L_{T}=32$} \\
\hline 0.26 & $0.28(1)$ & 0.33 & $1.06(1)$ & 0.42 & $1.917(6)$ & 0.54 & $2.90(1)$ \\
\hline 0.28 & $0.46(1)$ & 0.34 & $1.16(1)$ & 0.44 & $2.014(5)$ & 0.56 & $3.14(1)$ \\
\hline 0.29 & $0.56(1)$ & 0.36 & $1.419(10)$ & 0.46 & $2.116(5)$ & 0.58 & $3.401(9)$ \\
\hline 0.30 & $0.67(1)$ & 0.37 & $1.528(9)$ & 0.48 & $2.248(7)$ & 0.60 & $3.604(8)$ \\
\hline 0.31 & $0.78(1)$ & 0.38 & $1.632(8)$ & 0.50 & $2.428(8)$ & 0.62 & $3.805(7)$ \\
\hline 0.32 & $0.90(1)$ & 0.39 & $1.683(9)$ & 0.52 & $2.631(10)$ & 0.64 & $3.955(6)$ \\
\hline \multicolumn{8}{|c|}{$L_{T}=64$} \\
\hline 0.26 & $0.059(5)$ & 0.33 & $1.07(1)$ & 0.42 & $1.988(2)$ & 0.54 & $2.82(1)$ \\
\hline 0.28 & $0.20(1)$ & 0.34 & $1.23(1)$ & 0.44 & $2.000(1)$ & 0.56 & $3.21(1)$ \\
\hline 0.29 & $0.31(1)$ & 0.36 & $1.65(1)$ & 0.46 & $2.014(2)$ & 0.58 & $3.580(9)$ \\
\hline 0.30 & $0.51(2)$ & 0.37 & $1.772(9)$ & 0.48 & $2.048(4)$ & 0.60 & $3.844(7)$ \\
\hline 0.31 & $0.64(2)$ & 0.38 & $1.863(7)$ & 0.50 & $2.178(8)$ & 0.62 & $3.956(3)$ \\
\hline 0.32 & $0.86(1)$ & 0.39 & $1.918(6)$ & 0.52 & $2.45(1)$ & 0.64 & $3.994(2)$ \\
\hline \multicolumn{8}{|c|}{$L_{T}=128$} \\
\hline 0.26 & $0.002(1)$ & 0.33 & $1.059(5)$ & 0.44 & $1.999(1)$ & 0.54 & $2.81(1)$ \\
\hline 0.28 & $0.04(1)$ & 0.34 & $1.302(5)$ & 0.46 & $1.999(1)$ & 0.56 & $3.27(1)$ \\
\hline 0.29 & $0.06(1)$ & 0.36 & $1.87(1)$ & 0.48 & $2.001(1)$ & 0.58 & $3.84(1)$ \\
\hline 0.30 & $0.25(2)$ & 0.37 & $1.957(6)$ & 0.50 & $2.019(3)$ & 0.60 & $3.98(1)$ \\
\hline 0.32 & $0.84(1)$ & 0.39 & $1.992(4)$ & 0.52 & $2.24(1)$ & 0.64 & 4.0 \\
\hline \multicolumn{8}{|c|}{$L_{T}=256$} \\
\hline 0.26 & 0.00 & 0.31 & $0.42(5)$ & 0.36 & 2.00 & 0.54 & 2.91(1) \\
\hline 0.28 & 0.00 & 0.32 & $0.86(2)$ & 0.40 & 2.00 & 0.56 & $3.20(2)$ \\
\hline 0.29 & 0.00 & 0.33 & $1.02(1)$ & 0.50 & 2.00 & 0.58 & $3.97(1)$ \\
\hline 0.30 & $0.06(2)$ & 0.34 & $1.31(7)$ & 0.52 & $2.03(1)$ & 0.64 & 4.00 \\
\hline
\end{tabular}

TABLE V. Monte Carlo results for $\left\langle N_{f}\right\rangle$ at $U=0.3$ with open boundaries at selected values of $\mu$ and $L_{T}$ for $L_{X}=16$. These data are plotted in Fig. 13.

\begin{tabular}{|c|c|c|c|c|c|c|c|}
\hline$\mu$ & $\left\langle N_{f}\right\rangle$ & $\mu$ & $\left\langle N_{f}\right\rangle$ & $\mu$ & $\left\langle N_{f}\right\rangle$ & $\mu$ & $\left\langle N_{f}\right\rangle$ \\
\hline \multicolumn{8}{|c|}{$L_{T}=32$} \\
\hline 0.21 & $0.14(1)$ & 0.28 & $0.80(1)$ & 0.34 & $1.61(1)$ & 0.44 & $2.65(1)$ \\
\hline 0.22 & $0.21(1)$ & 0.29 & $0.94(1)$ & 0.36 & $1.83(1)$ & 0.46 & $2.89(1)$ \\
\hline 0.23 & $0.28(1)$ & 0.30 & $1.09(1)$ & 0.37 & $1.90(1)$ & 0.48 & $3.17(1)$ \\
\hline 0.24 & $0.36(1)$ & 0.31 & $1.22(1)$ & 0.38 & $1.99(1)$ & 0.50 & $3.43(1)$ \\
\hline 0.25 & $0.44(1)$ & 0.32 & $1.37(1)$ & 0.39 & $2.09(1)$ & 0.52 & $3.68(1)$ \\
\hline 0.26 & $0.55(1)$ & 0.33 & $1.49(1)$ & 0.42 & $2.39(1)$ & 0.54 & $3.91(1)$ \\
\hline \multicolumn{8}{|c|}{$L_{T}=64$} \\
\hline 0.22 & $0.04(1)$ & 0.30 & $1.13(2)$ & 0.38 & $2.002(3)$ & 0.48 & $3.26(1)$ \\
\hline 0.24 & $0.09(1)$ & 0.32 & $1.55(1)$ & 0.42 & $2.17(1)$ & 0.50 & $3.62(1)$ \\
\hline 0.26 & $0.27(1)$ & 0.34 & $1.83(1)$ & 0.44 & $2.46(1)$ & 0.52 & $3.86(1)$ \\
\hline 0.28 & $0.64(2)$ & 0.36 & $1.950(5)$ & 0.46 & $2.85(1)$ & 0.54 & $3.97(1)$ \\
\hline \multicolumn{8}{|c|}{$L_{T}=128$} \\
\hline 0.23 & 0.00 & 0.29 & $0.84(2)$ & 0.34 & $1.98(1)$ & 0.46 & $2.85(1)$ \\
\hline 0.26 & $0.07(1)$ & 0.30 & $1.15(3)$ & 0.35 & $1.99(1)$ & 0.48 & $3.31(2)$ \\
\hline 0.27 & $0.20(3)$ & 0.31 & $1.52(2)$ & 0.40 & 2.00 & 0.50 & $3.88(1)$ \\
\hline 0.28 & $0.46(3)$ & 0.32 & $1.83(1)$ & 0.44 & $2.24(1)$ & 0.54 & 4.00 \\
\hline \multicolumn{8}{|c|}{$L_{T}=256$} \\
\hline 0.25 & 0.0 & 0.30 & $1.19(4)$ & 0.42 & $2.00(1)$ & 0.48 & $3.32(2)$ \\
\hline 0.28 & $0.18(5)$ & 0.31 & $1.72(4)$ & 0.44 & $2.02(1)$ & 0.50 & $3.98(1)$ \\
\hline 0.29 & $0.89(3)$ & 0.34 & $1.99(1)$ & 0.46 & $2.87(2)$ & 0.52 & $3.99(1)$ \\
\hline
\end{tabular}

TABLE VI. Monte Carlo results for $\left\langle N_{f}\right\rangle$ at $U=0.3$ with open boundaries at selected values of $\mu$ and $L_{T}$ for $L_{X}=32$. These data are plotted in Fig. 13.

\begin{tabular}{lllccccc}
\hline \hline$\mu$ & $\left\langle N_{f}\right\rangle$ & $\mu$ & $\left\langle N_{f}\right\rangle$ & $\mu$ & $\left\langle N_{f}\right\rangle$ & $\mu$ & $\left\langle N_{f}\right\rangle$ \\
\hline \multicolumn{7}{c}{$L_{T}=32$} \\
0.22 & $0.67(2)$ & 0.28 & $1.87(2)$ & 0.36 & $3.64(2)$ & 0.44 & $5.40(2)$ \\
0.24 & $1.03(2)$ & 0.3 & $2.32(2)$ & 0.38 & $4.11(2)$ & 0.46 & $5.83(1)$ \\
0.26 & $1.43(2)$ & 0.34 & $3.22(2)$ & 0.4 & $4.52(1)$ & 0.48 & $6.29(1)$ \\
0.21 & $0.21(2)$ & 0.26 & $1.44(2)$ & 0.33 & $3.05(2)$ & 0.4 & $4.47(1)$ \\
0.22 & $0.35(2)$ & 0.28 & $1.90(2)$ & 0.35 & $3.54(2)$ & 0.42 & $4.93(1)$ \\
0.23 & $0.58(3)$ & 0.29 & $2.10(2)$ & 0.36 & $3.75(1)$ & 0.44 & $5.43(1)$ \\
0.24 & $0.82(2)$ & 0.31 & $2.50(2)$ & 0.37 & $3.92(1)$ & 0.46 & $5.85(1)$ \\
0.25 & $1.13(3)$ & 0.32 & $2.76(2)$ & 0.39 & $4.25(1)$ & 0.48 & $6.21(1)$ \\
\multicolumn{7}{c}{$L_{T}=128$} \\
0.21 & $0.019(6)$ & 0.26 & $1.71(2)$ & 0.33 & $3.04(3)$ & 0.4 & $4.25(2)$ \\
0.22 & $0.08(2)$ & 0.28 & $1.98(1)$ & 0.35 & $3.77(2)$ & 0.42 & $4.92(2)$ \\
0.23 & $0.25(4)$ & 0.29 & $2.03(2)$ & 0.36 & $3.92(2)$ & 0.44 & $5.64(2)$ \\
0.24 & $0.68(5)$ & 0.31 & $2.31(2)$ & 0.37 & $3.97(1)$ & 0.46 & $5.96(1)$ \\
0.25 & $1.27(5)$ & 0.32 & $2.67(3)$ & 0.39 & $4.06(1)$ & 0.48 & $6.057(9)$ \\
0.25 & $1.41(9)$ & 0.28 & $1.96(4)$ & 0.33 & $3.06(4)$ & 0.42 & $4.90(3)$ \\
0.26 & $1.91(5)$ & 0.31 & $2.06(2)$ & 0.36 & $3.97(3)$ & 0.44 & $5.87(3)$ \\
0.27 & $1.95(4)$ & 0.32 & $2.61(4)$ & 0.4 & $4.01(2)$ & 0.48 & $5.99(1)$ \\
\hline \hline
\end{tabular}

occurs will be this finite size fermion mass $m_{f}^{L_{X}}$. In order to see the effects of interactions, we plot $\left\langle N_{f}\right\rangle$ as a function of $\mu$ in the free theory (Fig. 12) and in the interacting theory with $U=0.3$ (Fig. 13), both with open boundary conditions. Selected data points have also been tabulated in Tables IV, V, and VI for benchmark purposes.

We study three different lattice sizes $L_{X}=12$ (left), $L_{X}=16$ (center), and $L_{X}=32$ (right). For each of these lattices, we study the effects of increasing $L_{T}$. Note that the critical value of $\mu$ where the first jump to $\left\langle N_{f}\right\rangle=2$ occurs shifts to lower values as $L_{X}$ increases in the free theory. We expect this value to vanish in the large $L_{X}$ limit since fermions are massless. However, in the interacting theory, we note the jump change in the critical value is smaller and should approach 0.183(1) (see Table I) as $L_{x}$ becomes large. Also, the jump becomes sharper as the anisotropy (value of $L_{T}$ ) is increased and approaches a step function as expected. To quantify, the value of $m_{f}^{L_{X}}$ we measure $\left\langle N_{f}\right\rangle$ for several values $\mu$ near the transition at two different values of $L_{T}$. In particular, with $L_{X}=12$, we use $L_{T}=64,128$, and with $L_{X}=32$, we use $L_{T}=128$, 256. We find the value of $\mu$ where $\left\langle N_{f}\right\rangle$ measured with different values of $L_{T}$ cross, using a linear fit near the crossing. These values of $\mu$ are taken to be estimates of $m_{f}^{12}$ and $m_{f}^{32}$. These numbers for different values of $U$ are tabulated in Table VII. Similarly, by fitting the chiral condensate susceptibility to the form

$$
\chi=\chi_{0}+B \mathrm{e}^{-m_{b}^{L_{X}} L_{T}},
$$


TABLE VII. The fermion and boson masses measured using the fermion number with open boundary conditions. The boson mass at $U=0$ is calculated directly from the free correlator on a finite lattice.

\begin{tabular}{lcclc}
\hline \hline$U$ & $m_{b}^{12}$ & \multicolumn{1}{c}{$m_{f}^{12}$} & \multicolumn{1}{c}{$m_{b}^{32}$} & \multicolumn{1}{c}{$m_{f}^{32}$} \\
\hline 0 & 0.17207 & $0.120(1)$ & 0.067393 & $0.045(5)$ \\
0.1 & $0.158(6)$ & $0.163(2)$ & $0.061(4)$ & $0.0705(6)$ \\
0.2 & $0.184(6)$ & $0.235(10)$ & $0.06(1)$ & $0.1397(3)$ \\
0.3 & $0.156(4)$ & $0.328(2)$ & $0.066(7)$ & $0.247(2)$ \\
0.4 & $0.143(4)$ & $0.425(2)$ & $0.060(3)$ & $0.356(1)$ \\
0.5 & $0.143(7)$ & $0.519(2)$ & $0.057(2)$ & $0.465(1)$ \\
0.6 & $0.137(1)$ & $0.601(2)$ & $0.049(5)$ & $0.556(1)$ \\
1.0 & $0.121(4)$ & $0.871(1)$ & $0.050(4)$ & $0.842(2)$ \\
$\infty$ & $0.114(4)$ & $\infty$ & $0.0476(9)$ & $\infty$ \\
\hline \hline
\end{tabular}

we can also extract the finite size boson mass $m_{b}^{L_{X}}$. These values are also given in Table VII for $L_{X}=12$ and 32. We find that, while $m_{f}^{L_{X}}$ increases sharply with $U$, $m_{b}^{L_{X}}$ decreases mildly.

\section{CONCLUSIONS}

In this work, we have studied the $1+1$-dimensional lattice Thirring model with staggered fermions at both zero and finite densities. We showed that the model is free of sign problems in the massless limit when open boundary conditions are used. In this case, we used the world line formulation to study the model. In the case of periodic and antiperiodic spatial boundary conditions, the sign problem is mild on square lattices but becomes severe when on asymmetric lattices. However, the fermion-bag formulation seems to alleviate the problem except at critical values of the chemical potential where fermion number jumps. We provide accurate estimates for the total particle number as a function of the chemical potential for a few lattice sizes. Our results could be used as a benchmark for future studies by other methods that attempt to solve the sign problem.

\section{ACKNOWLEDGMENTS}

We thank A. Alexandru and P. Bedaque for extensive discussions about their work and for providing their results so we can compare against our results on small lattices where such a comparison was possible. S. C. and J. R.'s work was supported by the U.S. Department of Energy, Office of Science, Nuclear Physics program under Award No. DE-FG02-05ER41368. V. A.'s work was supported by the U.S. Department of Energy under Grant No. DESC0010005.
[1] P. de Forcrand, Proc. Sci., LAT2009, 010 (2009).

[2] E. Seiler, in 35th International Symposium on Lattice Field Theory (Lattice 2017) Granada, Spain, 2017 (unpublished).

[3] M. Cristoforetti, F. Di Renzo, and L. Scorzato (AuroraScience Collaboration), Phys. Rev. D 86, 074506 (2012).

[4] A. Mukherjee, M. Cristoforetti, and L. Scorzato, Phys. Rev. D 88, 051502 (2013).

[5] Y. Tanizaki, Y. Hidaka, and T. Hayata, New J. Phys. 18, 033002 (2016).

[6] H. Fujii, S. Kamata, and Y. Kikukawa, J. High Energy Phys. 12 (2015) 125; 09 (2016) 172(E).

[7] J. Nishimura and S. Shimasaki, Phys. Rev. D 92, 011501 (2015).

[8] J. Bloch, J. Glesaaen, O. Philipsen, J. Verbaarschot, and S. Zafeiropoulos, Eur. Phys. J. Web Conf. 137, 07030 (2017).

[9] G. Aarts, E. Seiler, D. Sexty, and I.-O. Stamatescu, J. High Energy Phys. 05 (2017) 044.

[10] G. Aarts, F. Attanasio, B. Jger, and D. Sexty, J. High Energy Phys. 09 (2016) 087.

[11] A. Mukherjee and M. Cristoforetti, Phys. Rev. B 90, 035134 (2014).

[12] M. Cristoforetti, F. Di Renzo, A. Mukherjee, and L. Scorzato, Phys. Rev. D 88, 051501 (2013).

[13] G. Aarts and F. A. James, J. High Energy Phys. 08 (2010) 020.
[14] A. Alexandru, G. Basar, P. F. Bedaque, G. W. Ridgway, and N. C. Warrington, J. High Energy Phys. 05 (2016) 053.

[15] A. Alexandru, G. Basar, P. F. Bedaque, G. W. Ridgway, and N. C. Warrington, Phys. Rev. D 95, 014502 (2017).

[16] J. M. Pawlowski and C. Zielinski, Phys. Rev. D 87, 094509 (2013).

[17] D. Banerjee and S. Chandrasekharan, Phys. Rev. D 81, 125007 (2010).

[18] F. Bruckmann, C. Gattringer, T. Kloiber, and T. Sulejmanpasic, Phys. Rev. Lett. 115, 231601 (2015).

[19] A. Alexandru, P. Bedaque, H. Lamm, and S. Lawrence, Phys. Rev. D 96, 094505 (2017).

[20] E. Witten, Nucl. Phys. B145, 110 (1978).

[21] L. Del Debbio and S. Hands, Phys. Lett. B 373, 171 (1996).

[22] L. Del Debbio, S. J. Hands, and J. C. Mehegan (UKQCD Collaboration), Nucl. Phys. B502, 269 (1997).

[23] S. Chandrasekharan, Phys. Rev. D 82, 025007 (2010).

[24] S. Chandrasekharan and A. Li, Phys. Rev. Lett. 108, 140404 (2012).

[25] D. Li, Phys. Rev. D 94, 114501 (2016).

[26] H. G. Evertz, Adv. Phys. 52, 1 (2003).

[27] C. Gattringer, V. Hermann, and M. Limmer, Phys. Rev. D 76, 014503 (2007).

[28] U. Wolff, Nucl. Phys. B789, 258 (2008).

[29] C. Gattringer, T. Kloiber, and V. Sazonov, Nucl. Phys. B897, 732 (2015). 
[30] N. Prokof'ev and B. Svistunov, Phys. Rev. Lett. 87, 160601 (2001).

[31] O. F. Syljuåsen and A. W. Sandvik, Phys. Rev. E 66, 046701 (2002).

[32] M. Boninsegni, N. V. Prokof'ev, and B. V. Svistunov, Phys. Rev. E 74, 036701 (2006).

[33] S. Chandrasekharan and A. Li, Phys. Rev. Lett. 108, 140404 (2012).
[34] S. Chandrasekharan and A. Li, Phys. Rev. D 88, 021701 (2013).

[35] V. Ayyar and S. Chandrasekharan, Phys. Rev. D 93, 081701 (2016).

[36] D. J. Cecile and S. Chandrasekharan, Phys. Rev. D 77, 054502 (2008).

[37] B. B. Beard, R. J. Birgeneau, M. Greven, and U.-J. Wiese, Phys. Rev. Lett. 80, 1742 (1998). 